\title{
Front Matter: Volume 8604
}

, "Front Matter: Volume 8604," Proc. SPIE 8604, Nonlinear Frequency Generation and Conversion: Materials, Devices, and Applications XII, 860401 (28 March 2013); doi: 10.1117/12.2025040

SPIE. Event: SPIE LASE, 2013, San Francisco, California, United States 


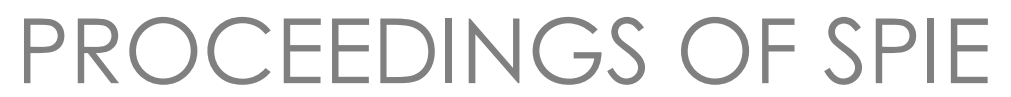

\section{Nonlinear Frequency Generation and Conversion: Materials, Devices, and Applications XII}

Konstantin L. Vodopyanov

Editor

5-7 February 2013

San Francisco, California, United States

Sponsored and Published by

SPIE 
The papers included in this volume were part of the technical conference cited on the cover and title page. Papers were selected and subject to review by the editors and conference program committee. Some conference presentations may not be available for publication. The papers published in these proceedings reflect the work and thoughts of the authors and are published herein as submitted. The publisher is not responsible for the validity of the information or for any outcomes resulting from reliance thereon.

Please use the following format to cite material from this book:

Author(s), "Title of Paper," in Nonlinear Frequency Generation and Conversion: Materials, Devices, and Applications XII, edited by Konstantin L. Vodopyanov, Proceedings of SPIE Vol. 8604 (SPIE, Bellingham, WA, 2013) Article CID Number.

ISSN: 0277-786X

ISBN: 9780819493736

Published by

SPIE

P.O. Box 10, Bellingham, Washington 98227-0010 USA

Telephone +1 3606763290 (Pacific Time) · Fax +1 3606471445

SPIE.org

Copyright (C) 2013, Society of Photo-Optical Instrumentation Engineers.

Copying of material in this book for internal or personal use, or for the internal or personal use of specific clients, beyond the fair use provisions granted by the U.S. Copyright Law is authorized by SPIE subject to payment of copying fees. The Transactional Reporting Service base fee for this volume is $\$ 18.00$ per article (or portion thereof), which should be paid directly to the Copyright Clearance Center (CCC), 222 Rosewood Drive, Danvers, MA 01923. Payment may also be made electronically through CCC Online at copyright.com. Other copying for republication, resale, advertising or promotion, or any form of systematic or multiple reproduction of any material in this book is prohibited except with permission in writing from the publisher. The CCC fee code is 0277-786X/13/\$18.00.

Printed in the United States of America.

Publication of record for individual papers is online in the SPIE Digital Library.

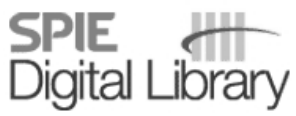

SPIEDigitallibrary.org

Paper Numbering: Proceedings of SPIE follow an e-First publication model, with papers published first online and then in print and on CD-ROM. Papers are published as they are submitted and meet publication criteria. A unique, consistent, permanent citation identifier (CID) number is assigned to each article at the time of the first publication. Utilization of CIDs allows articles to be fully citable as soon as they are published online, and connects the same identifier to all online, print, and electronic versions of the publication. SPIE uses a six-digit CID article numbering system in which:

- The first four digits correspond to the SPIE volume number.

- The last two digits indicate publication order within the volume using a Base 36 numbering

system employing both numerals and letters. These two-number sets start with 00, 01, 02, 03, 04, $05,06,07,08,09,0 A, 0 B \ldots 0 Z$, followed by 10-1Z, 20-2Z, etc.

The CID Number appears on each page of the manuscript. The complete citation is used on the first page, and an abbreviated version on subsequent pages. Numbers in the index correspond to the last two digits of the six-digit CID Number. 


\section{Contents}

ix Conference Committee

\section{SESSION 1 VISIBLE AND UV LASERS I}

860402 A narrow-band continuous-wave laser source at 191 nm (Invited Paper) [8604-1] M. Scholz, D. Opalevs, J. Stuhler, P. Leisching, W. Kaenders, TOPTICA Photonics AG (Germany);

G. Wang, X. Wang, R. Li, C. Chen, Technical Institute of Physics and Chemistry (China)

860403 Two photon absorption and stimulated Raman scattering in alkali vapor lasers [8604-2] J. E. Gallagher, E. J. Hurd, K. C. Brown, G. P. Perram, Air Force Institute of Technology (United States)

860404 Efficient concept generating $3.9 \mathrm{~W}$ of diffraction-limited green light with spectrally combined tapered diode lasers [8604-3]

A. Müller, O. B. Jensen, Technical Univ. of Denmark (Denmark); K.-H. Hasler, B. Sumpf,

G. Erbert, Ferdinand-Braun-Institut, Leibniz-Institut für Höchstfrequenztechnik (Germany);

P. E. Andersen, P. M. Petersen, Technical Univ. of Denmark (Denmark)

860405 Megawatt peak power UV microlaser [8604-4]

R. Bhandari, T. Taira, The Institute for Molecular Science (Japan)

\section{SESSION 2 VISIBLE AND UV LASERS II}

860406 Generation of tunable visible picosecond pulses by frequency-doubling of a quantum-dot laser in a PPKTP waveguide [8604-5]

K. A. Fedorova, The Univ. of Dundee (United Kingdom); G. S. Sokolovskii, The Univ. of Dundee (United Kingdom) and loffe Physico-Technical Institute (Russian Federation); D. I. Nikitichev, The Univ. of Dundee (United Kingdom); P. R. Battle, AdvR, Inc. (United States); D. A. Livshits, Innolume GmbH (Germany); E. U. Rafailov, The Univ. of Dundee (United Kingdom)

860408 Several watts compact CW green laser head without cooling by using PPMgSLT [8604-7] Y. Tomihari, S. Makio, M. Hoshi, M. Hatori, J. Hirohashi, K. Imai, H. Motegi, Y. Furukawa, Oxide Corp. (Japan)

860409 Pulsed picosecond $766 \mathrm{~nm}$ laser source operating between 1-80 MHz with automatic pump power management [8604-8]

T. Schönau, T. Siebert, R. Härtel, T. Eckhardt, D. Klemme, K. Lauritsen, R. Erdmann,

PicoQuant GmbH (Germany)

$8604 \mathrm{OA}$ Ultra-violet generation by third-harmonic conversion in conventional solid-core fibers [8604-9]

Y. Takushima, Y. Orii, A. Higashitani, T. Manabe, Spectronix Corp. (Japan) 
8604 OB High-power THz pulse generation and nonlinear THz spectroscopy (Invited Paper) [8604-10] K. Shinokita, H. Hirori, K. Tanaka, Kyoto Univ. (Japan); T. Mochizuki, C. Kim, H. Akiyama, The Univ. of Tokyo (Japan); L. N. Pfeiffer, K. W. West, Princeton Univ. (United States)

8604 OC Intense THz radiation produced in organic salt crystals for high-field applications (Invited Paper) [8604-11]

C. Vicario, C. Ruchert, Paul Scherrer Institut (Switzerland); C. P. Hauri, Paul Scherrer Institut (Switzerland) and Ecole Polytechnique Federale de Lausanne (Switzerland)

8604 OD Comparative study on THz time-domain spectroscopy using 780-nm 1.3-ps laser pulses with different detections of LT-GaAs photoconductive antenna and ZnTe electro-optic sampling [8604-12]

Y. Tadokoro, Y. Takida, Osaka City Univ. (Japan); H. Kumagai, Kitasato Univ. (Japan);

S. Nashima, A. Kobayashi, Osaka City Univ. (Japan)

8604 OE The widely tunable THz generation in QPM-GaAs crystal pumped by a near-degenerate dual-wavelength KTP OPO at around 2.127 $\mu \mathrm{m}$ [8604-13]

D. Xu, W. Shi, K. Zhong, Y. Y. Wang, P. Liu, J. Yao, Tianjin Univ. (China)

\section{SESSION 4 OPTICAL PARAMETRIC AND ULTRAFAST NONLINEAR DEVICES}

$8604 \mathrm{OH} \quad$ Narrow bandwidth tunable optical parametric generator [8604-17]

B. Dolasinski, P. Powers, Univ. of Dayton (United States)

8604 Ol Ho3+:LLF MOPA pumped RISTRA ZGP OPO at 3-5 $\mu \mathrm{m}$ [8604-18]

G. Stoeppler, M. Schellhorn, M. Eichhorn, Institut Franco-Allemand de Recherches de SaintLouis (France)

\section{SESSION $5 \quad$ NONLINEAR FIBER DEVICES AND APPLICATIONS}

8604 OK Widely tunable parametric generation of picosecond visible and mid-infrared radiation in optical fibers [8604-20]

A. Giree, C. Jauregui, Friedrich-Schiller-Univ. Jena (Germany); J. Limpert, Friedrich-SchillerUniv. Jena (Germany) and Helmholtz-Institute Jena (Germany); A. Tünnermann, FriedrichSchiller-Univ. Jena (Germany), Helmholtz-Institute Jena (Germany) and Fraunhofer Institute for Applied Optics and Precision Engineering (Germany)

$8604 \mathrm{OL}$ Polarization stabilization of vector solitons in circularly birefringent fibers induced by Raman effect [8604-21]

E. A. Kuzin, B. A. Villagomez-Bernabe, N. Korneev, B. Ibarra-Escamilla, A. Gonzalez-García, Instituto Nacional de Astrofísica, Óptica y Electrónica (Mexico); O. Pottiez, Ctr. de Investigaciones en Óptica, A.C. (Mexico); M. Duran-Sanchez, Univ. Tecnológica de Puebla (Mexico) 
$86040 \mathrm{~N}$ Experimental and theoretical investigations of single-frequency Raman fiber amplifiers operating at $1178 \mathrm{~nm}$ [8604-23]

I. Dajani, C. Vergien, Air Force Research Lab. (United States); B. Ward, U.S. Air Force

Academy (United States); C. Robin, S. Naderi, A. Flores, Air Force Research Lab. (United States); J.-C. Diels, The Univ. of New Mexico (United States)

SESSION 6 MID-IR FREQUENCY COMB AND SUPERCONTINUUM GENERATION

860400 Precision mid-infrared frequency combs and spectroscopic applications (Invited Paper) [8604-24]

D. Gatti, Politecnico di Milano (Italy); A. Castrillo, Seconda Univ. degli Studi di Napoli (Italy); A. Gambetta, T. Sala, G. Galzerano, P. Laporta, Politecnico di Milano (Italy); L. Gianfrani, Seconda Univ. degli Studi di Napoli (Italy); M. Marangoni, Politecnico di Milano (Italy)

8604 OR Multispectral mid-infrared imaging using frequency upconversion [8604-27]

N. Sanders, J. S. Dam, O. B. Jensen, P. Tidemand-Lichtenberg, C. Pedersen, Technical Univ. of Denmark (Denmark)

8604 OS High resolution mid-infrared spectroscopy based on frequency upconversion [8604-28] J. S. Dam, Q. Hu, P. Tidemand-Lichtenberg, C. Pedersen, Technical Univ. of Denmark (Denmark)

8604 OT Intracavity molecular spectroscopy in the mid-IR using ultra-broadband optical parametric oscillator [8604-29]

M. W. Haakestad, Stanford Univ. (United States) and Norwegian Defence Research Establishment (Nonway); T. P. Lamour, Stanford Univ. (United States) and Heriot-Watt Univ. (United Kingdom); N. Leindecker, A. Marandi, K. L. Vodopyanov, Stanford Univ. (United States)

\section{SESSION 7 NONLINEAR MATERIALS AND CHARACTERIZATION I}

8604 OV Progress in orientation-patterned GaP for next-generation nonlinear optical devices (Invited Paper) [8604-31]

V. Tassev, M. Snure, R. Peterson, K. L. Schepler, R. Bedford, M. Mann, Air Force Research Lab. (United States); S. Vangala, Solid State Scientific Corp. (United States); W. Goodhue, Univ. of Massachusetts Lowell (United States); A. Lin, J. Harris, M. Fejer, Stanford Univ. (United States); P. Schunemann, BAE Systems, Inc. (United States)

8604 OW Surface plasmon enhanced reflected second harmonic generation in periodically poled whispering gallery resonator [8604-32]

A. Saha, N. Goswami, P. Dey, National Institute of Technology Agartala (India)

$86040 Z$ Tapered nanowire waveguide layout for rapid optical loss measurement by 'cut-back' technique [8604-35]

M. F. A. Muttalib, R. Y. Chen, S. J. Pearce, M. D. B. Charlton, Univ. of Southampton (United Kingdom) 
860410 Tailorable stimulated Brillouin scattering in silicon nanophotonics (Invited Paper) [8604-36] P. T. Rakich, H. Shin, Yale Univ. (United States); W. Qiu, Massachusetts Institute of Technology (United States); R. Jarecki, J. A. Cox, R. H. Olsson III, A. Starbuck, Sandia National Labs. (United States); Z. Wang, Univ. of Texas at Austin (United States)

860412 Giant optical nonlinearity of graphene in a magnetic field [8604-38]

X. Yao, A. Belyanin, Texas A\&M Univ. (United States)

\section{POSTER SESSION}

860414 An efficient WDM-OTDM converter for spectrum interrogation based on nonlinear effects in electro-absorption modulator [8604-41]

C. Gao, Z. Wang, X. Qin, T. Wang, C. Ge, T. Yang, Tianjin Univ. (China)

860415 Terahertz generation in quasi-phase-matched GaAs wafers by pulse $\mathrm{CO}_{2}$ laser [8604-42] Z. Rao, Huazhong Univ. of Science and Technology (China) and Jiangxi Univ. of Traditional Chinese Medicine (China); X. Wang, D. Zuo, Huazhong Univ. of Science and Technology (China)

860416 Fourth-harmonic generation of the $\mathrm{CO}_{2}$ laser wavelength at $10.5910 \mu \mathrm{m}$ in $\mathrm{BaGa}_{4} \mathrm{~S}_{7}$ [8604-43]

K. Kato, Chitose Institute of Science and Technology (Japan) and Okamoto Optics Works, Inc. (Japan); T. Okamoto, T. Mikami, Okamoto Optics Works, Inc. (Japan); V. Petrov, MaxBorn-Institute for Nonlinear Optics and Ultrafast Spectroscopy (Germany); V. Badikov, D. Badikov, Kuban State Technological Univ. (Russian Federation); V. Panyutin, Max-BornInstitute for Nonlinear Optics and Ultrafast Spectroscopy (Germany)

860418 Parametric gain analysis in tellurite/phospho-tellurite hybrid microstructured optical fibers with an engineered chromatic dispersion [8604-45]

E. P. Samuel, H. T. Tong, Z. Duan, M. Liao, K. Asano, T. Suzuki, Y. Ohishi, Toyota Technological Institute (Japan)

8604 IF Tunable picosecond THz-wave generation based on trapezoidal MgO:LiNbO 3 crystal in novel pentagram-shaped pump-enhancement cavity [8604-53]

Y. Takida, Osaka City Univ. (Japan) and The Japan Society for the Promotion of Science (Japan); Y. Tadokoro, Osaka City Univ. (Japan); H. Kumagai, Osaka City Univ. (Japan) and Kitasato Univ. (Japan); S. Nashima, A. Kobayashi, Osaka City Univ. (Japan)

860411 Second-harmonic generation in $\mathrm{Hg}_{0.35} \mathrm{Cdd}_{0.65} \mathrm{Ga}_{2} \mathrm{~S}_{4}$ and $\mathrm{Hg}_{0.52} \mathrm{Cd}_{0.48} \mathrm{Ga}_{2} \mathrm{~S}_{4}$ [8604-56] K. Kato, Chitose Institute of Science and Technology (Japan) and Okamoto Optics Works, Inc. (Japan); T. Mikami, Okamoto Optics Works, Inc. (Japan); V. Petrov, Max-Born-Institute for Nonlinear Optics and Ultrafast Spectroscopy (Germany)

$86041 \mathrm{~J}$ Effects of nonlinear phase modulation on quantum frequency conversion using four-wave mixing Bragg scattering [8604-57]

L. Mejling, Technical Univ. of Denmark (Denmark); C. J. McKinstrie, Alcatel-Lucent Bell Labs. (United States); K. Rottwitt, Technical Univ. of Denmark (Denmark) 
8604 IK Nd:YAG laser pumped $\mathrm{HgGa}_{2} \mathrm{~S}_{4}$ parametric oscillator [8604-58]

K. Kato, Chitose Institute of Science and Technology (Japan); K. Miyata, MegaOpto, Co. Ltd. (Japan)

Author Index

Proc. of SPIE Vol. $8604860401-7$

Downloaded From: https://www.spiedigitallibrary.org/conference-proceedings-of-spie on 26 Apr 2023 Terms of Use: https://www.spiedigitallibrary.org/terms-of-use 
Proc. of SPIE Vol. $8604860401-8$

Downloaded From: https://www.spiedigitallibrary.org/conference-proceedings-of-spie on 26 Apr 2023 Terms of Use: https://www.spiedigitallibrary.org/terms-of-use 


\title{
Conference Committee
}

\author{
Symposium Chairs
}

Bo Gu, Bos Photonics (United States)

Andreas Tünnermann, Fraunhofer-Institut für Angewandte Optik und Feinmechanik (Germany) and Friedrich-Schiller-Universität Jena (Germany)

Symposium Cochairs

Friedhelm Dorsch, TRUMPF Werkzeugmaschinen GmbH + Co. KG (Germany)

Alberto Piqué, U.S. Naval Research Laboratory (United States)

Conference Chair

Konstantin L. Vodopyanov, Stanford University (United States)

Conference Cochair

Yehoshua Y. Kalisky, Nuclear Research Center Negev (Israel)

Conference Program Committee

Darrell J. Armstrong, Sandia National Laboratories (United States)

Pinhas Blau, Soreq Nuclear Research Center (Israel)

Majid Ebrahim-Zadeh, ICFO - Institut de Ciències Fotòniques (Spain)

Peter Günter, ETH Zurich (Switzerland)

Baldemar Ibarra-Escamilla, Instituto Nacional de Astrofísica, Óptica y

Electrónica (Mexico)

Yun-Shik Lee, Oregon State University (United States)

Rita D. Peterson, Air Force Research Laboratory (United States)

Peter Powers, University of Dayton (United States)

Peter G. Schunemann, BAE Systems (United States)

Kenneth L. Schepler, Air Force Research Laboratory (United States)

Andrei V. Shchegrov, KLA-Tencor Corporation (United States)

Wei Shi, Tianjin University (China)

Session Chairs

1 Visible and UV Lasers I

Andrei V. Shchegrov, KLA-Tencor Corporation (United States)

Yehoshua Y. Kalisky, Nuclear Research Center Negev (Israel) 
2 Visible and UV Lasers II

Andrei V. Shchegrov, KLA-Tencor Corporation (United States)

Yehoshua Y. Kalisky, Nuclear Research Center Negev (Israel)

3 Terahertz Generation

Peter Günter, ETH Zurich (Switzerland)

Wei Shi, Tianjin University (China)

4 Optical Parametric and Ultrafast Nonlinear Devices

Darrell J. Armstrong, Sandia National Laboratories (United States)

Peter E. Powers, University of Dayton (United States)

5 Nonlinear Fiber Devices and Applications

Wei Shi, Tianjin University (China)

Darrell J. Armstrong, Sandia National Laboratories (United States)

6 Mid-IR Frequency Comb and Supercontinuum Generation

Konstantin L. Vodopyanov, Stanford University (United States)

Kenneth L. Schepler, Air Force Research Laboratory (United States)

$7 \quad$ Nonlinear Materials and Characterization I

Peter G. Schunemann, BAE Systems (United States)

Baldemar Ibarra-Escamilla, Instituto Nacional de Astrofísica, Óptica y Electrónica (Mexico)

8 Nonlinear Materials and Characterization II

Peter G. Schunemann, BAE Systems (United States)

Baldemar Ibarra-Escamilla, Instituto Nacional de Astrofísica, Óptica y Electrónica (Mexico) 